\title{
Phase 1b Trial of LTI-01 (Single Chain Urokinase, scuPA) Intrapleural Fibrinolytic Therapy (IPFT) in Patients with Complicated Parapneumonic Effusions (CPE) or Empyema
}

Steven Idell ${ }^{1}$, Lutz Beckert ${ }^{2}$, Ben Brockway ${ }^{3}$, John Kolbe ${ }^{4}$, F. Graham Simpson ${ }^{5}$, John Wheatley ${ }^{6}$, Anne Marie Southcott ${ }^{7}$, YC Gary Lee ${ }^{8}$, Najb Rahman ${ }^{9}$, RW Light ${ }^{10}$, Steven Shoemaker ${ }^{11}$, John Gillies ${ }^{12}$, Andrey A. Komissarov ${ }^{1}$, Galina Florova ${ }^{1}$ and Krishna Sarva ${ }^{1}$

${ }^{1}$ Department of Cellular and Molecular Biology, The University of Texas Health Science Center at Tyler, Tyler, Texas, USA; ${ }^{2}$ University of Otago, Christchurch, New Zealand; ${ }^{3}$ University of Otago Dunedin School of Medicine, Dunedin, New Zealand; ${ }^{4}$ Auckland Hospital, Auckland, New Zealand; ${ }^{5}$ Cairns Hospital, Cairns, Australia; ${ }^{6}$ Westmead Hospital, Westmead, Australia; ${ }^{7}$ Footscray Hospital, Footscray, Australia; ${ }^{8}$ Sir Charles Gairdner Hospital, Perth, Australia; ${ }^{9}$ Nuffield Department of Medicine, University of Oxford, Oxford, UK; ${ }^{10}$ Department of Medicine, Vanderbilt University Medical Center, Nashville, Tennessee, USA;

${ }^{11}$ Nicosof LLC, Erie, Colorado, USA; ${ }^{12}$ Clinical Network Services (CNS), Auckland, New Zealand

Rationale: no dose-escalation trial has been performed to assess the safety, tolerability and preliminary efficacy of IPFT in patients with CPE/empyema. Unlike currently available IPFT, scuPA (LTI-01) generates durably active complexes that resist inhibition by PA inhibitor-1 (PAI-1).

Methods: This was an open-label, dose escalation safety trial (ANZCT Registry Trial ID: ACTRN12616001442493). The primary objective was to evaluate the safety and tolerability of LTI-01 IPFT. Subjects with CPE/empyema, loculation were initially treated with standard of care, including chest tube placement and antibiotics. If eligible subjects failed to drain after 3 hours of chest drain placement) LTI$01 \mathrm{IPFT}$ was started within 24 hours.t. Treatment was administered as a $30 \mathrm{ml}$ bolus through the chest tube with a dwell-time of 3 hours. Treatment was daily for up to 3 days. There were five dose escalation cohorts: 50,000 IU ; 100,000 IU; 200,000 IU; 400,000IU; (n=3 in these groups); and 800,000 IU (n=2). Secondary objectives were to evaluate pharmacokinetic (PK) and pharmacodynamic (PD) effects of escalating LTI-01 IPFT and to assess efficacy.

Results: Overall, LTI-01 was well tolerated with no safety signals of concern. There were no acute systemic bleeding events, and no treatment-emergent adverse events (TEAEs) were considered to be related to LTI-01. There were no consistent effects of treatment on systolic or diastolic blood pressure, respiratory rate or body temperature. A total of 22 TEAEs were recorded in 7 (50.0\%) of the 14 subjects. There were two serious AEs that were felt to be unrelated to LTI-01. One patient in the 50,000 IU group died of metastatic carcinoma at day 20. Another treated with 400,000 IU died of a ruptured lung abscess at day 86. There was no treatment-related systemic fibrinogenolysis. scuPA antigen was consistently elevated in pleural fluids at 3 hours after LTI-01 treatment (PD, $p<0.01$ ) but did not increase in plasma. Decreased pleural opacities were seen in all but the one LTI-01-treated subject with a malignant pleural effusion. Mean leukocyte/neutrophil counts and CRP levels decreased from day 2 (pre-dose) to days 7-28. Both patients receiving $800,000 \mathrm{IU}$ required only two doses in order to achieve drainage and relief of pleural sepsis.

Conclusions: At the doses tested, LTI-01 IPFT was well-tolerated and safe. No local or systemic bleeding was observed. LTI-01 appeared to be effective in reducing pleural collection in patients with CPE and empyema. Suggestions of efficacy were observed following administration of LTI-01 IPFT.

Support: Lung Therapeutics, Inc. (LTI), NIH SMARTT HHSN268201100014C(SI) and NIH UO-1 HL12184101A1(SI). 